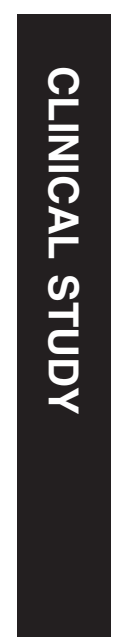

\title{
Surgical treatment of 32 cases of long- term atopic keratoconjunctivitis using the amniotic membrane
}

${ }^{1}$ Tianjin Medical University Eye Hospital, Tianjin, China

${ }^{2}$ Department of Ophthalmology, Columbia University, New York, NY, USA

${ }^{3}$ Beijing Minzhong Eye Hospital, Beijing, China

${ }^{4}$ Department of Ophthalmology, Shin Kong Wu Ho-Su Memorial Hospital, Fu-Jen Catholic University, Taipei, Taiwan

Correspondence: X-r Li, Tianjin Medical University Eye Hospital, No.251 Fu Kang Road Nankai District, Tianjin 300384, China

Tel: + 8622 23346430;

Fax: + 862223346434

E-mail: xiaorli@163.com

Received: 8 January 2013 Accepted in revised form: 3 July 2013

Published online: 16 August 2013

\begin{abstract}
Purpose To evaluate the use of surgical treatment with amniotic membrane for longterm atopic keratoconjunctivitis. Damaged corneas were repaired with various techniques: amniotic membrane transplantations, amniotic membrane coverings, amniotic membrane fillings (AMFs), and amniotic membrane inlay fillings, the latter of which were combined with glycerol-preserved corneal transplants. Methods This retrospective study was conducted on 37 eyes belonging to 37 patients with atopic keratoconjunctivitis. Thirty-two patients were classified into four groups according to surgical technique. Five patients undergoing medical management served as controls. Surgical outcome was measured by recovery time and long-term visual improvement.

Results In all surgical eyes, integrity of ocular tissues was effectively restored and symptoms were reduced at $24.4 \pm 13$ days post recovery. Mean best-corrected visual acuity improved from $0.6 \pm 0.2$ to $0.198 \pm 0.16$ logarithm of the minimum angle of resolution $(P<0.001)$. There were no intraoperative or postoperative complications, with the exception of two recurring cases, both controlled by medication. Recovery time of the control groups lasted $52 \pm 16$ days. In controls, mean best-corrected visual acuity improved from $0.74 \pm 0.15$ to $0.54 \pm 0.29$ logarithm of the minimum angle of resolution $(P \leq 0.05)$. The vision improvement has significant difference for surgical treatment $v s$ medical. (Mann-Whitney $U$-test, $U=119, P<0.05$, one tailed).Vision improvements remained stable during a mean follow-up period of $21.7 \pm 3.8$ months.
\end{abstract}

J Yang ${ }^{1,2}$, F-h Yang ${ }^{3}$, C-H Peng ${ }^{2,4}$, D Erol ${ }^{2}$, SH Tsang ${ }^{2}$ and $\mathrm{X}-\mathrm{r} \mathrm{Li}{ }^{1}$
Conclusion Patients suffering from severe chronic atopic keratoconjunctivitis and its complications can benefit from suitable surgical treatments: transplants, covers, fillings, or corneal graft surgeries supplemented with AMFs.

Eye (2013) 27, 1254-1262; doi:10.1038/eye.2013.161; published online 16 August 2013

Keywords: amniotic membranes; atopic keratoconjunctivitis; transplantation; corneal ulcer and perforation

\section{Introduction}

Atopic keratoconjunctivitis (AKC) is a perennial, relatively serious form of allergic keratoconjunctivitis that is often observed in atopic individuals. ${ }^{1}$ AKC is characterized by chronic inflammation of the conjunctiva and by various related corneal epithelial pathologies, including superficial punctate keratopathy, epithelial defects, shield ulcers, and even corneal perforations. When corneal lesions caused by AKC persist over time, complications develop, including scarring, corneal vascularization, amblyopia, and eventually reduced visual acuity.

AKC-related corneal lesions pose great challenges for treatment. These lesions are more resistant to therapy than other ocular allergies, especially as the condition advances and complications begin to appear. Potential treatments must be chosen with care; anti-allergic eye drops are the first line of treatment. If these are shown to be ineffective, then steroid eye drops are added. In addition, any development of atopic blepharitis may require immediate treatment with a prescription-strength oral 
steroid. ${ }^{2}$ In its advanced form, the severe corneal lesions caused by the disease may require surgery.

Since the 1940s, the amniotic membrane had been discussed as a promising source of graft tissue for corneal and conjunctival reconstructive surgeries. The utility of amniotic tissue has been established by several clinical trials; it has proven useful in many clinical situations, including the treatment of acute burns, corneal epithelial defects, and conjunctival scarring. In these surgeries, the membrane is used as a graft or patch to cover and repair the cornea. ${ }^{3}$ Amniotic tissue is thought to shorten healing time and promote re-epithelialization.

Corneal graft transplantation is another type of surgery commonly used to treat corneal ulcers or perforations, when these are serious enough that the cornea must be replaced. Corneal grafts have the ability to restore integrity of the eye and promote visual recovery. ${ }^{4}$ However, over time, the nutrients in the tissue dwindle, and, in many cases, postoperative immunological rejection has been reported after corneal graft surgery. 5

Corneal grafts can be improved by reducing inflammation and promoting integration of graft tissue in the eye. To this end, the amniotic membrane has established anti-inflammatory and anti-vascular properties and has been shown to facilitate the migration of epithelial cells. ${ }^{3}$ However, amniotic tissues do no not provide sufficient structural support to be used by itself for many types of surgeries. ${ }^{6}$ For this study, we combined the advantages of corneal graft and amniotic membrane graft surgeries in one subgroup of patients, using both methods to maximize surgical treatment of the cornea.

Not more than a few surgical attempts have been reported in the past to treat severe AKC. In this study, we performed a retrospective review of 37 cases (62 eyes) of AKC patients treated over the past 5 years. Thirty-two of these eyes were treated surgically with amniotic membrane transplantations (AMTs), coverings, or fillings, or with a combination of corneal path grafts and amniotic tissue fillings. To better describe how to treat $\mathrm{AKC}$ patients in need of surgical intervention, we present long-term outcomes of AKC patients who have been treated with amniotic grafts or preserved corneal path grafts. Patients were assigned to surgeries based on the features of their cases.

\section{Materials and methods}

This study was approved by the Institutional Review Board of Tianjin Medical University Eye Hospital in Tianjin, China. Over a period between 2005 and 2012, a total of 37 subjects ( 25 male, 12 female; ages between 5 and 61 years, mean 37.97 years) with a confirmed diagnosis of AKC were recruited. AKC was diagnosed based on the following features, as described by Guglielmetti and colleagues: ${ }^{7}$ the disease is associated with other atopic conditions (rhinitis, eczema, or asthma), occurs at any time point in the course of associated atopic disease, and is combined with evidence of corneal involvement, independent of degree of severity of atopic disease.

These 37 cases had a long history of misdiagnoses and mismanagement. They had responded poorly to conventional anti-allergic treatments. Typical treatments were potent mast cell stabilizers and low-dose corticosteroids: $0.1 \%$ olopatadine hydrochloride ophthalmic solution twice per day (Patanol; Alcon Laboratories, Fort Worth, TX, USA), $0.2 \%$ cyclosporine A eye drops 4 times a day, tobramycin/dexamethasone eye drops 4-6 times per day (Tobradex; Alcon Laboratories), and artificial tears 4 times per day. In some cases, a subconjunctival injection of dexamethasone was added twice per week. However, most of corneas still displayed severe resistant epithelial defects, erosions, or ulcerations (Table 1) more than 1 month into treatment. We offered surgical intervention for these cases. Thirty-two subjects provided written informed consent to take part in the study after an explanation of the nature, risks, and possible adverse consequences of the procedures. Five patients who chose not to undergo surgical treatment, but instead were treated with medication, served as control group.

Human amniotic membrane and preserved corneal tissue were used for these treatments. AM and glycerolpreserved corneal patch grafts were prepared as previously described. ${ }^{8,9}$ All membranes and grafts were screened by blood serum testing to exclude the human immunodeficiency virus, hepatitis virus type $B$, hepatitis virus type $C$, and syphilis.

In this study, cases involving recurrent and refractory corneal epithelial defection were treated by AMT. The procedure was as follows: a single layer of AM equivalent in size to the corneal epithelial defect was selected and sutured along the defection border with the membrane stromal side facing down. Cases featuring recurrent corneal erosions were treated with amniotic membrane covering (AMC). The membrane surface was sutured to cover the entire corneal surface extending beyond the limbus. Surgical debridement was performed before the tissue covering was set in place. A single layer of membrane with stromal side down was secured to the conjunctiva with a running 10-0 nylon suture.

Fillings were used for small perforations. When a corneal ulcer with perforation was evident, and the size of ulcer and microperforation were not $>1.5 \mathrm{~mm}$, we used the amniotic membrane to fill in the gaps in tissue left behind by the ulcer and microperforation. Three 


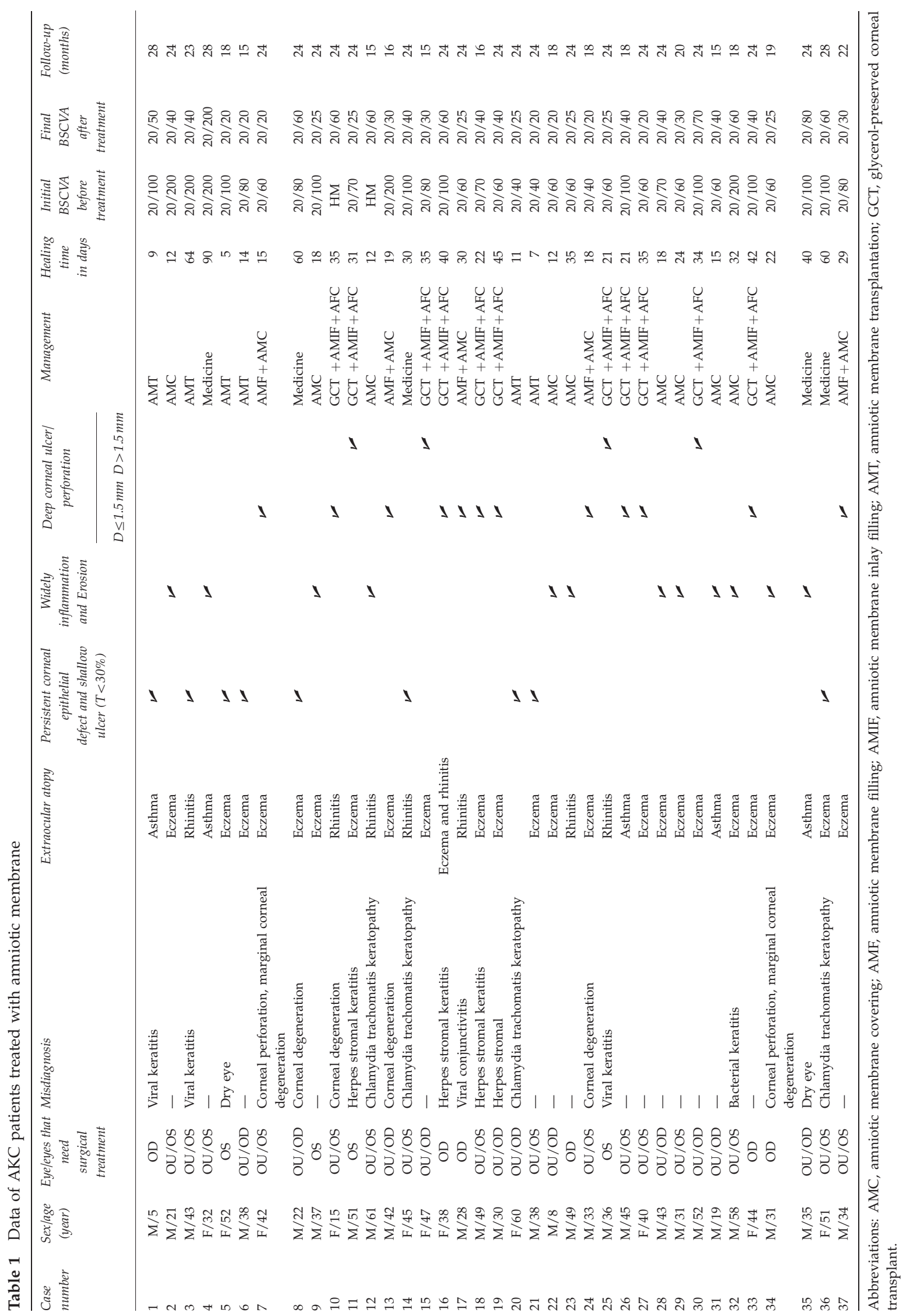


star-shaped sutures were used to ensure that the AM filled the holes in the cornea tightly. Another single sheet of amniotic membrane with a larger perimeter than the wound was used to cover the filling, allowing it to heal.

Alternatively, in corneas with perforations of $>1.5 \mathrm{~mm}$ in size, graft corneal transplants and amniotic membrane filling (AMF) were performed in quick succession. Donated glycerol-preserved corneal grafts were placed into sterile saline for recovery. The grafts were then fitted to the size of the perforation and placed in the eye of the host in the deep stromal layer secured in position by edge to edge sutures. The amniotic membrane was used to fill in the space between the corneal graft and a top lager layer of AMC, as needed. This larger layer of amniotic membrane was selected to cover the whole area with the defect. Tissue was trimmed to fit the shape of the ulcer and sutured with interrupted 10-0 nylon sutures.

After the surgery, all patients were treated with antiallergic and anti-inflammation medications for durations of at least 2 weeks. Doses of the medication were adjusted at follow-up. For these follow-up appointments, patients were asked to return at 1, 2, and 4 weeks postoperatively, and then at 3, 12, and 24 months. In addition, patients returned whenever any recurrent episodes occurred. At each postoperative visit, each patient underwent a complete ocular examination with imaging by slit-lamp photography. The period of recovery, as determined by reduction of ocular inflammation, the recovery of corneal epithelium, the recovery of anterior chamber, and the reconstruction of cornea, was recorded in days.

If healing was completed within 3 months, the surgery was classed as a short-term or primary success. If longterm follow-up found no recurrence of inflammation, an absence of postoperative corneal melts, no persistent epithelial defects, and significant and sustained restoration of vision, the surgery was classed as a long-term or secondary success.

\section{Results}

Table 1 displays each patient's clinical information. Thirty-seven subjects took part in the study. Twenty of these were misdiagnosed before their first visit to our hospital (Figure 1). Period of misdiagnosis varied between 30 days and 3 years.

As some of our patients had been misdiagnosed for long periods, corneal scarring, corneal opacity, and vascularization of the cornea were commonly observed. Some patients exhibited severe central, marginal or shield ulcers, or even corneal perforation. All patients displayed a variety of atopic conditions with various

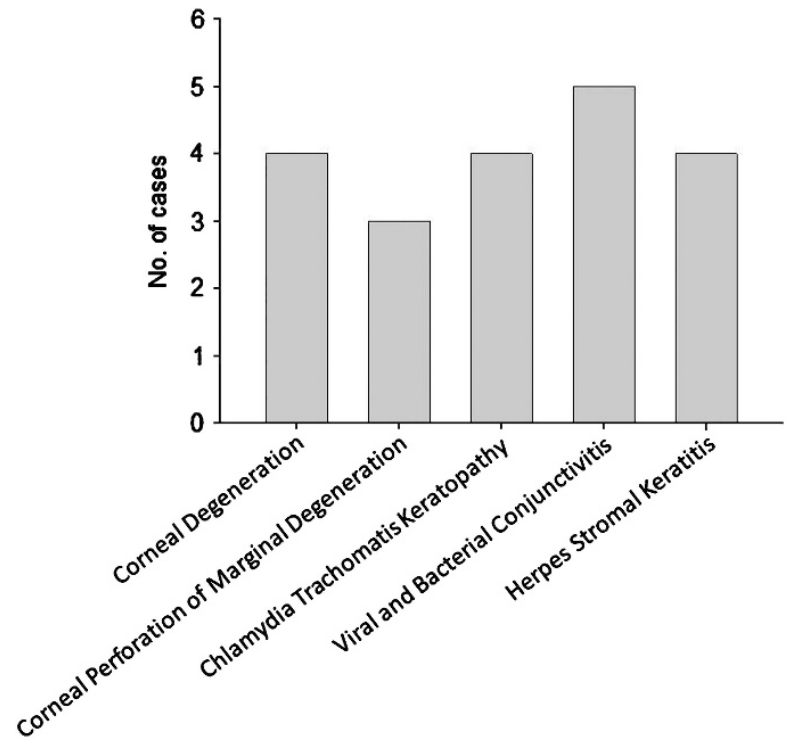

Figure 1 Misdiagnoses by condition and number of cases.

Table 2 Patient characteristics

\begin{tabular}{lc}
\hline Patient characteristics & $\begin{array}{c}\text { Number of patients } \\
(\mathrm{n}=37)\end{array}$ \\
\hline $\begin{array}{l}\text { Mean age (years) at presentation } \\
\text { (range) }\end{array}$ & $37.97(5-61)$ \\
$\mathrm{M} / \mathrm{F}$ & $25 / 12$ \\
Bilateral/single & $27 / 10$ \\
Surgical treatment & 32 (one eye on one \\
& patient) \\
Mean follow-up (range) (months) & $21.7 \pm 3.8(15-28)$ \\
Extraocular atopy & $37(100 \%)$ \\
Allergic rhinitis & 13 \\
Eczema & 22 \\
Asthma & 6 \\
Family history of atopy & 7 \\
\hline
\end{tabular}

degrees of severity: allergic rhinitis, eczema, and asthma (Table 2).

All patients were followed up for $21.7 \pm 3.8$ months. The time required for the AM to be reabsorbed varied from 1 week to 1 month. Generally, the inner layer remained attached until the ulcer and perforation healed. Because of irregular astigmatism and residual scarring in different parts of cornea, the best-corrected visual acuity was uneven in different groups (Table 3). In the surgical groups, two patients experienced the recurrence of AKC, manifesting as itching, foreign-body sensation, decreases in vision, and signs of formation of new shield ulcers at the edges of the transplanted AM, but these were controlled by the topical medications $0.1 \%$ olopatadine and $0.05 \%$ cyclosporine A eye drops twice a day without a need for further surgery. As all surgical cases healed in $24.4 \pm 13$ days, and vision had improved stably by the time of long-term follow-up visits, all surgeries were 
Table 3 Outcomes of treatment group

\begin{tabular}{|c|c|c|c|c|c|c|}
\hline Group & State of cornea before treatment & $\begin{array}{c}\text { Eyes } \\
\text { (number) }\end{array}$ & Treatment type & $\begin{array}{l}\text { Recovery } \\
\text { time } \\
\text { (days) }\end{array}$ & $\begin{array}{l}\text { BSCVA, before } \\
\text { treatment } \\
\text { (logarithm) }\end{array}$ & $\begin{array}{l}\text { BSCVA, after } \\
\text { treatment } \\
\text { (logarithm) }\end{array}$ \\
\hline 1 & Persistent corneal epithelial defect & 6 & AMT & $8.8 \pm 3.3$ & $0.6 \pm 0.27$ & $0.16 \pm 0.07$ \\
\hline 2 & $\begin{array}{l}\text { Severe widespread superficial corneal } \\
\text { inflammation and erosion and shallow ulcer } \\
\text { (thick }<30 \% \text { ) }\end{array}$ & 10 & AMC & $19.45 \pm 8$ & $0.56 \pm 0.23$ & $0.2 \pm 0.13$ \\
\hline 3 & $\begin{array}{l}\text { Deep corneal ulcer and microperforation } \\
(\mathrm{d} \leqslant 1.5 \mathrm{~mm})\end{array}$ & 5 & $\mathrm{AMF}+\mathrm{AMC}$ & $22 \pm 6.8$ & $0.57 \pm 0.26$ & $0.17 \pm 0.08$ \\
\hline 4 & $\begin{array}{l}\text { Deep corneal ulcer and microperforation } \\
(\mathrm{d}>1.5 \mathrm{~mm})\end{array}$ & 11 & $\mathrm{GCT}+\mathrm{AMIF}+\mathrm{AMC}$ & $34 \pm 7.8$ & $0.58 \pm 0.1$ & $0.24 \pm 0.17$ \\
\hline $\begin{array}{l}\text { Total } \\
\text { surgical } \\
\text { cases }\end{array}$ & - & 32 & - & $24.4 \pm 13$ & $0.6 \pm 0.2$ & $0.198 \pm 0.16$ \\
\hline $\begin{array}{l}\text { Control } \\
\text { group }\end{array}$ & $\begin{array}{l}\text { Persistent corneal epithelial defect or Severe } \\
\text { corneal erosion and shallow ulcer }\end{array}$ & 5 & Medical & $52 \pm 16$ & $0.74 \pm 0.15$ & $0.54 \pm 0.29$ \\
\hline
\end{tabular}

classed as both primary and secondary successes. Mean best-corrected visual acuity improved from $0.6 \pm 0.2$ to $0.198 \pm 0.16$ logarithm of the minimum angle of resolution $(P<0.001, t=5.8034)$.

The recovery time of the control group was $52 \pm 16$ days, much longer than that of the surgery group. In one case, new corneal lesions appeared on the original area of corneal lesions 3 months into treatment (Figure 2, control case). Mean best-corrected visual acuity improved from $0.74 \pm 0.15$ to $0.54 \pm 0.29$ logarithm of the minimum angle of resolution $(P \leq 0.05, t=4.298)$. The vision improvement has significant difference for surgical treatment $v s$ medical. (Mann-Whitney $U$-test, $U=119, P=0.044$, one tailed).

\section{Surgical case examples}

Case 1 A 58-year-old farmer with a history of 2 years of bilateral eye redness, itching, and swelling, and with decreased vision in the left eye, was referred for evaluation. On initial examination, best-corrected visual acuity was 20/40 OD and 20/200 OS. External examination revealed eczema of each eyelid with thickened lid margins. Slit-lamp examination revealed punctate epithelial inflammation on the temporal side of the right eye. There was a 2-mm white area of inflammation with notable new vascular invasion on the superior area of the left eye and extensive epithelial erosions (Figure 2, case 1a). After multiple AKC medications were administered over 3 weeks, 20/20 visual acuity in the right eye was attained, but a central superficial ulcer of about $4 \mathrm{~mm}$ appeared on the left eye (Figure 2, case 1b). We classed this case with group 2 and performed an AMC surgery on the ulcer in the left eye (Figure 2, case 1c). Corneal inflammation was controlled at 3-week follow-up. Vision (20/60) and corneal epithelialization were found on examination 18 months after the surgery (Figure 2, case 1d).

Case 2 A 43-year-old man had suffered from photophobia and tearing in both eyes for a period of 1 year. Over the 2 months before his visit to the hospital, this had been accompanied by decreased vision in the left eye. Before enrolling in the study, he had previously been misdiagnosed with viral keratitis and was treated with antiviral eye drops for more than 1 month. After 1 month of treatment with the antiviral medication, hyperemia of the conjunctiva developed in both eyes. The cornea of the left eye was swollen, with a corneal flake ulcer evident in the upside of the left eye (Figure 2, case 2a), in addition to intensive neovascularization in the vicinity of the ulcer. His total serum IgE level was $5000 \mathrm{IU} / \mathrm{ml}$. After a definite diagnosis was made, the patient was treated and kept under medication for 1 week. The conjunctival inflammation was greatly reduced, but the corneal lesion remained unchanged. In addition, he had developed a resistant corneal ulcer in the upside of the left eye. His best-corrected visual acuity was 20/25 in the right eye and 20/200 in the left eye. Because of refractory corneal involvement, he underwent surgery with an AMT in the left eye (group 1) (Figure 2, case 2b).

Twenty days after the procedure, AM grafts showed excellent maintenance. However, 40 days after the surgery, neovascularization was evident around the corneal lesion and under the membrane (Figure 2, case 2c). New shield ulcers formed again on the edge of original ulcer at 50 days after treatment (Figure 2, case $2 \mathrm{~d}$ ). Giving a recent history, the patient revealed that he had reduced the medicine shortly after the surgery; following this, doses of topical medication were 


\section{Control case}

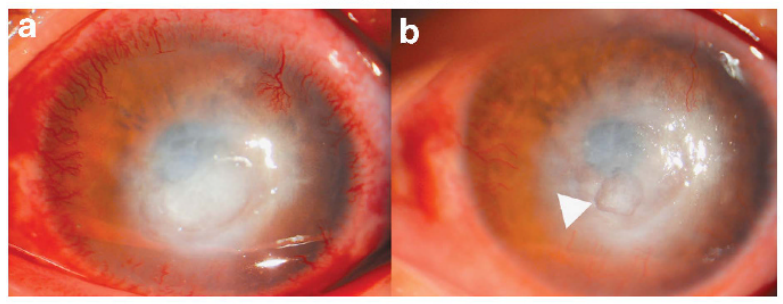

Surgical case 1

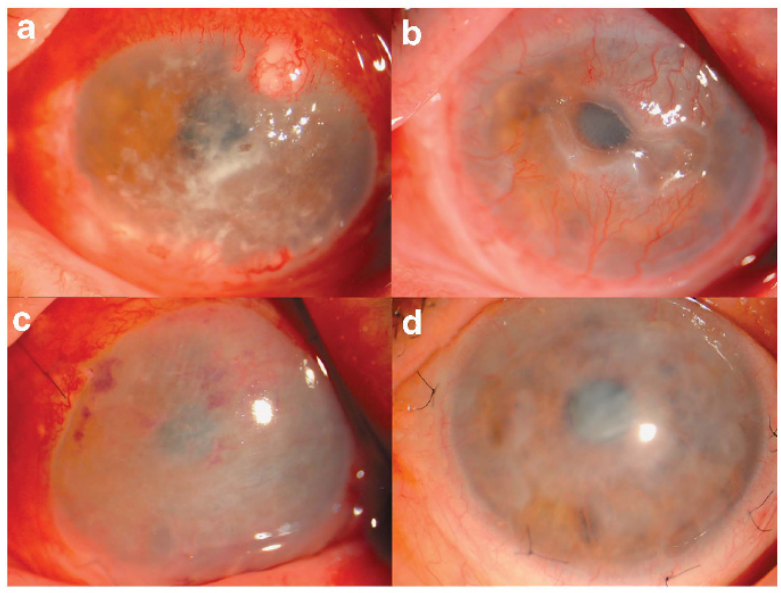

Surgical case 3

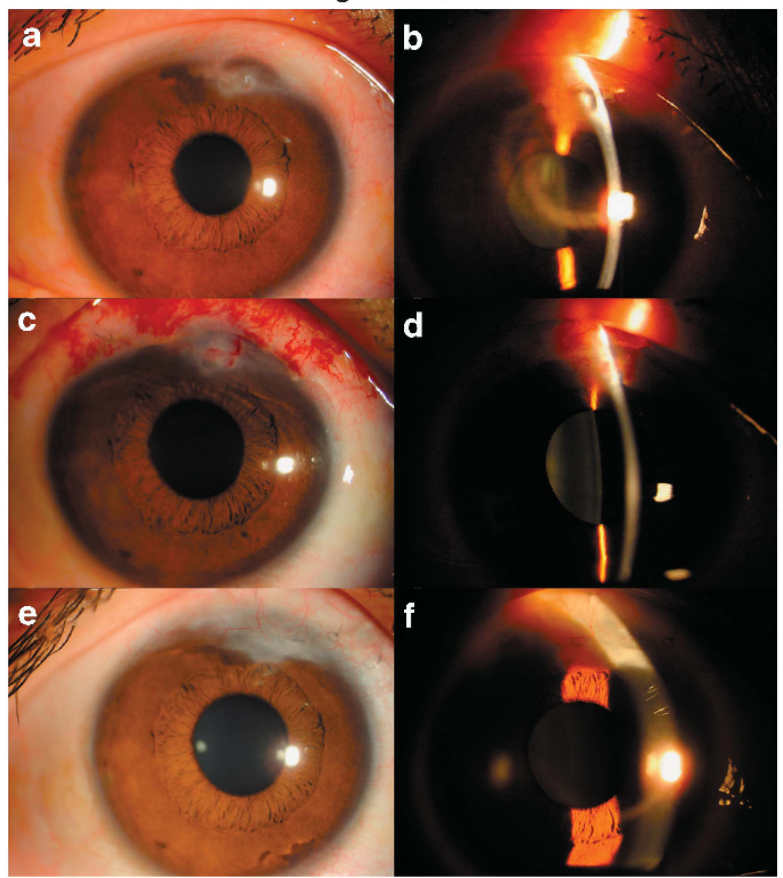

\section{Surgical case 2}

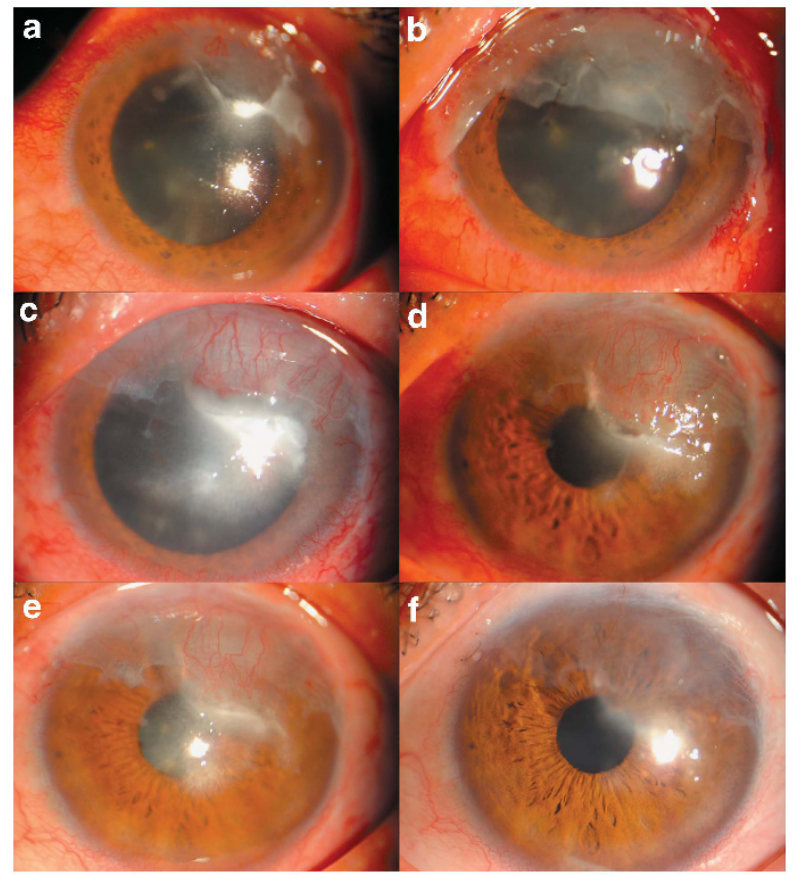

\section{Surgical case 4}

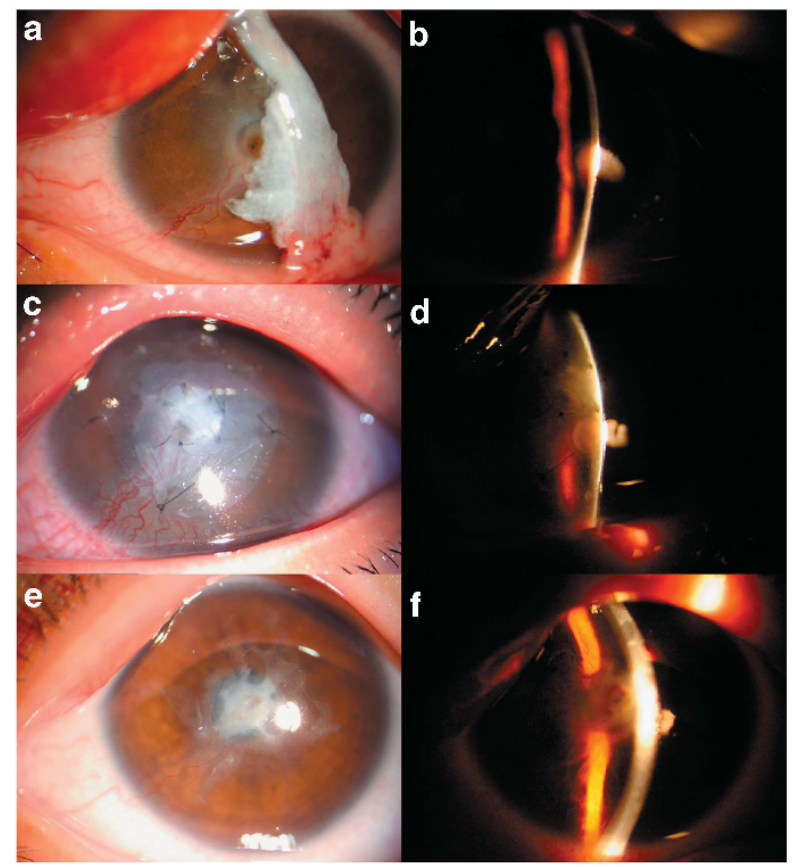

Figure 2 Control case and surgical case examples. Control case a: severe corneal swelling is present in the central area and corneal epithelial defects are apparent, with peripheral superficial vascular invasion. Control case b: vascular invasion and swelling are both reduced after 3 months of medical treatment, but a new 2-cm ulceration area (arrow) appears again on the original epithelial defect area. Surgical cases: case 1 underwent AMC surgery; case 2 underwent AMT Surgery; case 3 underwent combined AMC and AMF surgery; and case 4 underwent combined GCT, AMIF, and AMC surgeries. 
administered again, and, after 2 weeks, corneal swelling was reduced. The patient continued taking the medication at its prescribed dose for 5 months, until the cornea was clear and all vascularization had disappeared (Figure 2, case 2e). The best-corrected vision of the patient was restored to 20/40 after 10 months (Figure 2, case $2 \mathrm{f})$. No reoccurrence was reported at 2-year follow-up.

Case 3 A 28-year-old man complained of 2 years of photophobia and itching in his right eye. Prior diagnosis was dry eye. The patient appeared in the emergency room with red eye and ocular pain in the right eye, reporting that vision had decreased dramatically in the past 20 days. Slit-lamp examination showed a marginal corneal perforation of $1 \mathrm{~mm}$ in diameter at the 1230 clock position, with the iris bulging (Figure 2, cases $3 a$ and $b$ ). The patient had no history of trauma. A combined AMF with AMC surgery (group 3) was performed (Figure 2, cases $3 c$ and $d$ ). The perforation healed in 30 days.

At 2-year follow-up, his vision had improved to 20/25 from the initial 20/60 (Figure 2, cases 3e and f).

Case 4 A 15-year-old female student was hospitalized for 2 weeks in another hospital because of photophobia and tearing that had both persisted for more than 1 year. Vision in the left eye decreased suddenly one day while the patient was washing her face. Diagnosis showed corneal degeneration in both eyes, with a central corneal perforation of the left eye. Conjunctival flap surgery had already been performed on the patient, but the corneal lesion did not improve after the treatment. At this time, the patient was referred to our hospital. Vision measured 20/20 OD and hand motion OS. Slit-lamp examination revealed lid swelling and redness in the bulbar and palpebral conjunctivas of both eyes. Cornea epithelial defects spanned a 3-mm area of inflammation with a superficial vascular invasion on the left eye. The conjunctival flap was detached, covering the center of the cornea of the left eye, with a 3-mm central perforation and extensive neovascularization in the perforation area. The anterior chamber had disappeared (Figure 2, cases $4 \mathrm{a}$ and b). Laboratory tests showed a high level of $\operatorname{IgE}$ $(6120 \mathrm{IU} / \mathrm{ml})$ in the patient's serum. Local anesthesia was administered, and the flap was removed with a spatula. We then treated this case as a member of group 4, with combined surgeries: glycerol-preserved corneal transplant (GCT), amniotic membrane inlay filling (AMIF), and AFC (Figure 2, cases 4c and d). The anterior chamber had re-formed 1 week after surgery. At 35 days, the corneal perforation was substantially healed. Visual acuity in the right eye had improved to 20/60 at 24-month follow-up (Figure 2, cases 4e and f). Visual acuity had not completely recovered because of irregular astigmatism and residual scarring. No reoccurrence was reported at the 2-year follow-up.

\section{Discussion}

This study showed that even in eyes with refractory $\mathrm{AKC}$, in which long-term immunological injuries had led to corneal damage because of misdiagnosis and mismanagement, appropriate surgical treatment could still result in significant, visible improvements of the corneal surface. Among all subjects treated with surgical intervention, only two recurrences were observed, both cases in which patients stopped the medication on their own. In addition, corneal epithelial erosions, ulcers, and perforations healed sooner after the surgery compared with the control group.

Chronic inflammation caused by AKC can be easily mistaken for other conditions and misdiagnosed (Figure 1). Disease mismanagement can allow the disease to worsen, making it subsequently harder to treat. Most cases in this study involved refractory corneal complications even after multiple medicinal treatments.

The surgical option was considered to avoid sightthreatening corneal sequelae. Surgical treatments with amniotic membrane tissue were tailored to the patients' symptoms. From our results, it is clear that although recovery is possible, once corneal deep ulcer and perforation have formed, the recovery time generally lengthens (Table 3). A correct AKC diagnosis and a suitable surgery involvement should be given as early as possible.

The lessons learned from these cases provide several key points to making a diagnosis. First, most patients have a history of atopic conditions, such as allergic rhinitis, eczema, and asthma dermatitis. Disease course is usually chronic, with most patients reporting more than half a year of history. Testing for serum IgE levels may help confirm the diagnosis. Vernal keratoconjunctivitis is another form of allergic keratoconjunctivitis involving high serum IgE, but is distinguished by its pattern of seasonally exacerbation.

It is well established that amniotic membrane has many properties that make it favorable for transplant surgeries: an avascular stromal matrix, antiinflammatory, and anti-scarring properties, and the ability to enhance epithelialization, and so on. ${ }^{10}$ Persistent and complicated corneal lesions are common in AKC patients. The treatment of AKC by AM transplantation has been discussed in a few case reports. Two case reports described successful corneal epithelialization after long-term corneal epithelial defects were treated with single AMT. ${ }^{11,12}$ After AM transplantation, long-term corneal epithelial defects can heal dramatically. Some reports showed that for the smaller-sized $(<1.5 \mathrm{~mm})$ corneal ulcer and perforations, 
multiple layers of AM transplant surgeries have been used to treat the cornea with the stromal side facing the anterior chambers. ${ }^{13,14}$ Multiple layers of AM not only rapidly epithelialized these small defects but also reduced ocular inflammation and resulted in recovery of corneal stromal thickness. As all of the surgical treatments of our 32 patients involved AM, and all featured different degrees of corneal ulcers or perforations, we have combined these features in a single report.

AMF and inlay filling are variations on a single approach: using multilayer AM to treat AKC in cases without any perforated ulcers or with smaller-sized perforations. Nonetheless, we would argue that a more exact placement of AM across corneal lesions would have improved outcomes in the cornea in this study. The differences between the AM fillings and multilayer AM transplants are that the former can allow the AM to completely fill the inside of the ulcer, bringing the AM and ulcer into direct contact and raising the surface area on which AM can have a direct effect. All the six patients who were treated by AMF and AMC healed relatively quickly, within 1 month, with no evidence of recurrence at 2-year follow-up.

For AKC cases featuring corneal perforations $>1.5 \mathrm{~mm}$, corneal path grafting may be considered. Rodriguez-Ares et $a l^{13}$ have reported that the success rate of multilayer AM transplant surgery depends on perforation size: when corneal perforations are $>1.5 \mathrm{~mm}$, there is only a $40 \%$ chance that surgery will succeed. In addition, earlier surgeries have shown that corneal path grafts are suitable for cases with corneal perforations and severe corneal ulcers, ${ }^{15}$ effectively restoring the integrity of the eye.

Such findings suggest that for larger perforations, corneal path grafts should be the first choice for treatment. However, patients with AKC and high serum IgE levels are thought to pose increased risks of graft rejection. ${ }^{16}$ For this reason, we used glycerol-preserved corneal patches to treat AKC patients. Glycerolpreserved corneal tissue, in contrast to fresh tissue, is thought to lack antigen-presenting cells, and thus to be unable to directly sensitize the recipient $\mathrm{T}$ cells or lead to activation of an indirect immune transplant rejection pathway. This method could prevent allograft rejection and promote graft survival rate in high-risk corneas. ${ }^{9}$ Applying $0.2 \%$ cyclosporine A eye drops after surgery may also maintain graft health, ${ }^{17}$ as well as decrease the recurrence rate of $\mathrm{AKC}$.

Recently, a novel AMIF technique was used to treat necrotic scleral calcification. ${ }^{6}$ Inlay fillings were found to aid graft survival. We used the same methods combined with graft corneal transplant on 11 patients, testing whether AMIFs and coverings would stabilize the graft and promote healing. All patients showed efficient healing and no recurrences.
On the whole, surgical treatments using the tailored amniotic membrane may be highly effective for patients suffering from AKC. The disease is the most severe of all forms of ocular atrophy and may result in poor vision for many patients. Although clearly earlier correct diagnosis is needed, and therapy requires a multidisciplinary approach, for a subset of chronic AKC patients, surgical treatment may become an ultimate necessity. AM tissue, a source of nutrients with anti-inflammatory proprieties, not only can be used as an effective graft tissue but also may promote postsurgical recovery. Finally, AMCs, AM inlay fillings, and GCTs surgeries can be combined with highly effective results.

\section{Summary}

What was known before

- Amniotic membrane (AM) transplantation as a treatment for corneal ulcer and perforation.

- AM inlay filling technology applied on necrotic scleral calcification treatment.

What this study adds

- Summerized virous AM transplantation on the treatment of corneal complication of atopic keratoconjunctivitis.

- AM inlay filling technology applied on cornea treatment.

\section{Conflict of interest}

The authors declare no conflict of interest.

\section{References}

1 Rachdan D, Anijeet DR, Shah S. Atopic keratoconjunctivitis: present day diagnosis. Br J Ophthalmol 2012; 96(11): 1361-1362.

2 Sakarya Y, Sakarya R. Treatment of refractory atopic blepharoconjunctivitis with topical tacrolimus $0.03 \%$ dermatologic ointment. J Ocul Pharmacol Ther 2012; 28(1): 94-96.

3 Kruse FE, Cursiefen C. Surgery of the cornea: corneal, limbal stem cell and amniotic membrane transplantation. Dev Ophthalmol 2008; 41: 159-170.

4 Vanathi M, Sharma N, Titiyal JS, Tandon R, Vajpayee RB. Tectonic grafts for corneal thinning and perforations. Cornea 2002; 21(8): 792-797.

5 Rahman I, Carley F, Hillarby C, Brahma A, Tullo AB. Penetrating keratoplasty: indications, outcomes, and complications. Eye (Lond) 2009; 23(6): 1288-1294.

$6 \mathrm{Kim} \mathrm{BH}$. Surgical treatment of necrotic scleral calcification using combined conjunctival autografting and an amniotic membrane inlay filling technique. Eye (Lond) 2011; 25(11): 1484-1490.

7 Guglielmetti S, Dart JK, Calder V. Atopic keratoconjunctivitis and atopic dermatitis. Curr Opin Allergy Clin Immunol 2010; 10(5): 478-485. 
8 Prabhasawat P, Barton K, Burkett G, Tseng SC. Comparison of conjunctival autografts, amniotic membrane grafts, and primary closure for pterygium excision. Ophthalmology 1997; 104(6): 974-985.

9 Li J, Yu L, Deng Z, Wang L, Sun L, Ma H et al. Deep anterior lamellar keratoplasty using acellular corneal tissue for prevention of allograft rejection in high-risk corneas. Am J Ophthalmol 2011; 152(5): 762-770.e3.

10 Seitz B, Das S, Sauer R, Mena D, Hofmann-Rummelt C. Amniotic membrane transplantation for persistent corneal epithelial defects in eyes after penetrating keratoplasty. Eye (Lond) 2009; 23(4): 840-848.

11 Takano Y, Fukagawa K, Miyake-Kashima M, Tanaka M, Asano-Kato N, Dogru M et al. Dramatic healing of an allergic corneal ulcer persistent for 6 months by amniotic membrane patching in a patient with atopic keratoconjunctivitis: a case report. Cornea 2004; 23(7): 723-725.

12 Fukuda K, Yamada N, Nishida T. Case report of restoration of the corneal epithelium in a patient with atopic keratoconjunctivitis resulting in amelioration of ocular allergic inflammation. Allergol Int 2010; 59(3): 309-312.

13 Rodriguez-Ares MT, Tourino R, Lopez-Valladares MJ, Gude F. Multilayer amniotic membrane transplantation in the treatment of corneal perforations. Cornea 2004; 23(6): 577-583.

14 Prabhasawat P, Tesavibul N, Komolsuradej W. Single and multilayer amniotic membrane transplantation for persistent corneal epithelial defect with and without stromal thinning and perforation. Br J Ophthalmol 2001; 85(12): 1455-1463.

15 Jhanji V, Young AL, Mehta JS, Sharma N, Agarwal T, Vajpayee RB. Management of corneal perforation. Surv Ophthalmol 2011; 56(6): 522-538.

16 Power WJ, Tugal-Tutkun I, Foster CS. Long-term follow-up of patients with atopic keratoconjunctivitis. Ophthalmology 1998; 105(4): 637-642.

17 Ghoraishi M, Akova YA, Tugal-Tutkun I, Foster CS. Penetrating keratoplasty in atopic keratoconjunctivitis. Cornea 1995; 14(6): 610-613. 